\begin{tabular}{|c|l|}
\hline Title & Flagellar waveforms of gametes in the brown alga Ectocarpus silicul osus \\
\hline Author(s) & Kinoshita, Nana; Shiba, Kogiku; Inaba, Kazuo; Fu, Gang; Nagasato, Chikako; Motomura, Taizo \\
\hline Citation & $\begin{array}{l}\text { European journal of phycology, 51(2), 139-148 } \\
\text { https://doi.org/L0.1080/09670262.2015.1109144 }\end{array}$ \\
\hline Issue Date & 2016-05 \\
\hline Doc URL & http://hdl.handle.net/2115/65238 \\
\hline Rights & $\begin{array}{l}\text { This is an A ccepted Manuscript of an article published by Taylor \& Francis in European Journal of Phycology on } \\
\text { O5/2016, available online: http://wwww.tandfonline.com/10.1080/09670262.2015.1109144. }\end{array}$ \\
\hline Type & article (author version) \\
\hline File Information & Kinoshita_et_al.pdf \\
\hline
\end{tabular}

Instructions for use 


\section{Flagellar Waveforms of gametes in the brown alga Ectocarpus siliculosus}

Nana Kinoshita ${ }^{1}$, Kogiku Shiba ${ }^{2}$, Kazuo Inaba ${ }^{2}$, Gang Fu ${ }^{3}$, Chikako Nagasato ${ }^{3}$ and Taizo Motomura $3^{3 *}$

${ }^{1}$ Graduate School of Environmental Science, Hokkaido University, Sapporo 060-0810, Hokkaido, Japan

${ }^{2}$ Shimoda Marine Research Center, University of Tsukuba, Shimoda 415-0025, Shizuoka, Japan

${ }^{3}$ Muroran Marine Station, Field Science Center for Northern Biosphere, Hokkaido University, Muroran 051-0003, Hokkaido, Japan

*Corresponding author: Taizo Motomura

Email: motomura@fsc.hokudai.ac.jp

Tel: +81 143222846

Fax: +81 143224135

A running title: Heterogeneous flagella in brown algae 


\section{Abstract}

Brown algae are members of the Stramenopiles and their gametes generally have two heterogenous flagella: a long anterior flagellum (AF) with mastigonemes, and a short posterior flagellum (PF). In this study, swimming paths and flagellar waveforms in free-swimming and thigmotactic-swimming male and female gametes, and male gametes during chemotaxis, were quantitatively analysed in the model brown alga Ectocarpus siliculosus. This was performed using a high-speed video camera. It was revealed that AF plays a role in changing the locomotion of male and female gametes from free-swimming to thigmotactic-swimming, and also in changing the swimming path of male gametes from linear to circular during chemotaxis. In the presence of a sex pheromone, male gametes changed their swimming path from linear (swimming path curvature, $0-0.02 \mu \mathrm{m}^{-1}$ ) to middle and small circular path (swimming path curvature, $0.04-0.20 \mu \mathrm{m}^{-1}$ ). The flagellar asymmetry and the deflection angle of the AF became larger, whereas the oscillation pattern of the AF was stable. However, there was no correlation between those of the AF and the path curvature when the male gametes showed middle to small circular paths. PF irregularly changed the deflection angle, and the oscillation pattern was unstable depending on the gradient of the sex pheromone concentration. AF waveforms were independent of PF locomotion during chemotaxis. It is quantitatively suggested that the AF has the ability to change the swimming path of male gametes-for example, from a highly linear path to a circular path-while changes in locomotion from a middle circle path to a small circle path is the result of beating of the PF. 
Key words: anterior flagellum, brown algae, chemotaxis, flagellar movement, gametes, posterior flagellum, thigomotaxis 


\section{Introduction}

Motile gametes of the brown algae usually have two heterogeneous flagella, a long anterior flagellum (AF) and a short posterior flagellum (PF) (Clayton, 1989; O' Kelly, 1989; Anderson, 2004). The AF is decorated with mastigonemes and generates a force for moving forward of gametes (Jahn et al., 1964; Holwill \& Sleigh, 1967; Bouck, 1969). The PF frequently has a basal swelling part (paraflagellar body), and provides rapid lateral beats for changing the direction of the cell movement (Kawai \& Kreimer, 2000; Maier, 1995).

Chemotactic responses of male gametes to a sex pheromone from settled female gametes have been studied for many years in the isogamous brown alga, Ectocarpus siliculosus (Dillwyn) Lyngbye (Müller \& Falk, 1973; Müller, 1978; Geller \& Müller, 1981). First, female gametes shortly settle on the substratum and secrete the sex pheromone ‘ectocarpen’ (Müller, 1967, Müller et al., 1971). Second, free-swimming male gametes began to swim in close contact with the surface of slide glass or cover slip. This response is enhanced by the presence of the sex pheromone from the female gamete, and is regarded as thigmotaxis (Müller, 1978; Geller \& Müller, 1981). The swimming velocity of male gametes decreased, and the swimming radius became smaller. Third, the male gamete changes the AF waveform, which causes the cell body to move in circular paths under existence of the sex pheromone. This behaviour of the male gamete is regarded as chemokinesis (Maier, 1993, 1995). PF of the male gamete performs fast, unilateral beating, when it detects a decrease of the sex pheromone concentration gradients. This PF behaviour results in a reorientation towards the 
pheromone source and is defined as chemoklinotaxis (Maier, 1993, 1995). Afterwards, the male gamete anchors itself with the tip of the AF on the surface of the female gamete, and finally, the male gamete fuses to the female one (Müller \& Falk, 1973). Therefore, the AF and PF of male gametes have crucial roles in approach and contact with female gametes.

Geller \& Müller (1981) firstly reported that the AF of male gametes in $E$. siliculosus perform asymmetric bending in the presence of the sex pheromone, which shows a negative linear correlation between the average of deflection angle of the AF from the cell axis and the radius of the track of the male gametes during chemokinesis. Their analysis was based on measuring the deflection of the first visible bend of the AF from the cell axis. However, information on the flagellar waveform of brown algae has been limited compared to the case of metazoa (Gibbons, 1981; Inaba, 2003) and the green alga, Chlamydomonas (Mitchell, 2000). Analyses of flagellar waveforms in sperm of marine invertebrates using high-speed camera revealed that flagellar waveforms became asymmetric when sperm changed the swimming direction toward the sex pheromone source. Their flagellar waveforms remained symmetric when sperm swam directly toward the pheromone source (Miller \& Brokaw, 1970; Miller, 1975; Miller, 1977). It has been reported that $\mathrm{Ca}^{2+}$ was a primary factor regulating the symmetry and asymmetry of flagellar waveforms (Kaupp et al., 2008; Yoshida \& Yoshida, 2011).

In the present study, swimming paths and two heterogeneous flagellar waveforms of male and female gametes of the brown alga E. siliculosus were quantitatively analysed using a high-speed camera, focusing on free- and thigmotactic-swimming male 
and female gametes and chemotactic-swimming male gametes to understand the more precise relationship between $\mathrm{AF}$ and $\mathrm{PF}$ waveforms of male gametes during fertilization in brown algae.

\section{Materials and methods}

Preparation of gametes

Male and female gametophytes (strain Ec32m and Ec25f) of Ectocarpus siliculosus (Dillw.) Lyngbye were gifts from Drs Peters, A. F. (Bezhin Rosko, France) and Coelho, S. M. (UMR 7139 CNRS-UPMC, Station Biologique de Roscoff, France). These gametophytes were cultured in the half strength PES medium (Provasoli, 1968) under cool white fluorescent lamps $\left(30-40 \mu \mathrm{mol} \mathrm{m}^{-2} \mathrm{~s}^{-1}\right)$ at $15{ }^{\circ} \mathrm{C}$ in long day conditions $(14 \mathrm{hr}$ light / 10 hr dark). Two days after changing to new medium, gametes were released from plurilocular gametangia within $2 \mathrm{hr}$ of light irradiation. They showed a strong negative phototaxis and gathered at the opposite side of culture dishes from the light source.

Observations and recording

Thirty $\mu \mathrm{l}$ of culture medium containing freshly liberated male and female gametes was added in an observation chamber (90 $\mu$ m depth). This observation chamber was made of double-faced adhesive tape between a slide glass and a cover slip. Images of flagellar waveforms were recorded with a phase contrast microscope (BX51, Olympus, Tokyo, Japan) with a 40× objective (UPlan FLN, Olympus) connected to a high-speed CCD 
camera (HAS220, Ditect, Tokyo, Japan) at 200 or 600 fps (frames per sec) and 1/1000 sec shutter speed. Observations were carried out using an R-58 red cut-off glass filter (S-058, HOYA, Tokyo, Japan) in the dark room at room temperature (ca. $20^{\circ} \mathrm{C}$ ), for preventing the effect of blue light on phototaxis of E. siliculosus (Kawai et al., 1990). Image analyses of flagellar waveforms were executed only on planner bending waveforms.

\section{Data analysis}

Swimming velocities, swimming path curvatures, flagellar curvatures and the deflection angle of gametes were analysed by Bohboh software (Bohboh Soft, Tokyo, Japan). Images of the cell body of gametes were automatically tracked, and the swimming velocities were measured along the zigzag trajectory of the cell bodies during flagellar beating cycles (trajectory swimming velocity). The path curvature, which is the reciprocal of the radius of curvature $(\rho)$ of the circular swimming path, was also calculated (Fig. S1). Trajectories in a clockwise direction were defined as a plus and trajectories in a counterclockwise direction were defined as a minus. The flagellar waveforms of gametes were automatically traced. The flagellar curvature, which is the reciprocal of the radius of curvature (r) of the flagellar waveform, was calculated based on the method of Baba and Mogami (1985). Flagellar asymmetry was expressed as the ratio of the maximal curvature of $\mathrm{P}$ bend (Principal bend) and that of $\mathrm{R}$ bend (Reverse bend) ( $\mathrm{P}$ bend Max / $\mathrm{R}$ bend Max). The ratio should be equal to one when the flagella show completely symmetric bending, and is should be greater than one when they show 
an asymmetric waveform. This ratio is referred to as the asymmetric index (Shiba et al., 2008).

The angle of the tangent to the flagellar shaft was measured with respect to the direction of the axis of the cell body. It was referred to the deflection angle (Fig. S2). The right side was defined as plus and the left side was defined as minus, when the AF was localized to the direction of forward movement and viewed from the ventral side of the gametes. The anterior end of cell body was defined as zero with respect to the deflection angle of the AF and the posterior end of cell body was defined as zero in with respect to the deflection angle of the PF. The points which tended to take on large values in maximum and minimum of the deflection angle was $8 \mu \mathrm{m}$ from the tip of the AF and $1 \mu \mathrm{m}$ from the tip of the PF.

\section{Results}

Locomotion of free- and thigmotactic-swimming gametes

Two patterns of swimming were observed in male and female gametes of E. siliculosus. The first observed pattern was that the gametes freely swam with helical rotations of the cell body without touching anything. Trajectories of these gametes showed spiral or undulating lines (Fig. 1). The second observed pattern was that they swam just on the substratum by eliminating the helical rotation of the cell bodies. These thigmotactic-swimming male and female gametes followed straight to large circular paths (Fig. 2). 
Just after adding $30 \mu \mathrm{l}$ of culture medium containing freshly liberated male or female gametes to a chamber, about $20 \%$ of gametes showed thigmotaxis and $80 \%$ of gametes freely swam. After three min, about $50 \%$ of gametes showed thigmotactic-swimming. The number of thigmotactic-swimming gametes gradually increased.

The swimming velocity was $205.2 \pm 49.5 \mu \mathrm{m} \mathrm{s}^{-1}(\mathrm{n}=95)$ in free-swimming male gametes and $131.5 \pm 24.1 \mu \mathrm{m} \mathrm{s}^{-1}(\mathrm{n}=115)$ in thigmotactic-swimming gametes, and there were a significant difference $(\mathrm{P}<0.001)$ (Table 1) between the two values. In female gametes, the swimming velocity was $191.0 \pm 48.4 \mu \mathrm{m} \mathrm{s}^{-1}(\mathrm{n}=90)$ in free-swimming gametes and $151.8 \pm 38.6 \mu \mathrm{m} \mathrm{s}^{-1}(\mathrm{n}=115)$ in thigmotactic-swimming gametes. There was also a significant difference $(\mathrm{P}<0.001)$ between these values. No significant difference was observed between the swimming patterns of male and female gametes.

The path curvature, which is the reciprocal number of the radius of curvature $(\rho)$ of the circular-swimming gametes, was calculated. Trajectories in a clockwise rotation were defined as plus and those in a counterclockwise direction were defined as minus (Fig. S1). Thigmotactic-swimming male gametes showed highly linear to large circular paths without a pheromone source, and $66 \%$ of the path curvature values ranged from $-0.02-0.02 \mu m^{-1}(n=115)$ (Fig. 3). Similar tendencies were also observed in female gametes.

AF movements of free- and thigmotactic-swimming gametes 
Flagellar waveform of free- and thigmotactic-swimming male and female gametes, which showed typical swimming velocities and path curvatures, were quantitatively analysed. The AF showed periodic oscillations, but the PF showed only irregular oscillations in Ectocarpus gametes. Therefore, flagellar curvature, asymmetric index and beat frequency were analysed only in the AF.

AF movements of free-swimming gametes were composed of symmetric and asymmetric waveforms (Figs 4-6, 10, Movie S1). The AF of thigmotactic-swimming gametes usually showed asymmetric waveforms (Figs 7-9, 11, Movie S2). In the case of free-swimming male gametes, which showed the highly linear paths (path curvature $\left.-0.01-0.009 \mathrm{~m}^{-1}\right)(\mathrm{n}=5)$, maximum values of P-bend and R-bend in the AF were 1.13 \pm 0.09 and $1.08 \pm 0.06$, respectively. The asymmetric index ( $\mathrm{P}$ bend / $\mathrm{R}$ bend) of the AF was $1.05 \pm 0.03$ as the whole region (Fig. 12, S3). The slightly curved free-swimming male gametes $\left(>0.012 \mu \mathrm{m}^{-1}\right)$ had a asymmetric waveform which was similar to those of thigmotactic-swimming gametes (not shown). The AF of thigmotactic-swimming male gametes, which showed highly linear paths (from -0.008 to $0.008 \mu \mathrm{m}^{-1}, \mathrm{n}=7$ ), displayed asymmetric waveforms (Fig. 12). The maximum values of P-bend and R-bend were $1.16 \pm 0.13$ and $0.98 \pm 0.12$, respectively (Fig. S4). Next, we separately measured the asymmetric index at the base, middle and tip regions of AF (Fig. 12) for examining which part of AF showed a conspicuous asymmetry. The asymmetric indices were $1.05 \pm 0.07$ at the base $(0-5 \mu \mathrm{m}), 1.19 \pm 0.045$ at the middle $(6-10 \mu \mathrm{m}), 1.42 \pm$ 0.15 at the tip $(11-14 \mu \mathrm{m})$, and $1.24 \pm 0.075$ as the whole region $(0-14 \mu \mathrm{m})$. The AF waveform of these two types of gametes, which showed highly linear paths, were 
significantly different in asymmetric index, especially between the tip region and the whole region $(\mathrm{P}<0.001)$ (Fig. 12). Female gametes also showed similar patterns of AF waveforms. The asymmetric waveform in AF was observed in both free- and thigmotactic-swimming gametes; however, the symmetric waveform in the AF was observed only in free-swimming gametes during straight swimming.

The deflection angle between the flagellum and the axis of cell body was obtained. The average values of the deflection angle in the AF of free- and thigmotactic-swimming male gametes, which showed a highly linear path, were $6.3^{\circ} \pm$ $8.0^{\circ}\left(\max 102.6^{\circ}\right.$, $\left.\min 80.8^{\circ}\right)$ and $-22.9^{\circ} \pm 4.5^{\circ}\left(\max 45.2^{\circ}\right.$, min $\left.-106.6^{\circ}\right)$, respectively (Table 1, Fig. 13). There was a statistically significant difference between these two values ( $\mathrm{P}<0.001)$. The deflection angle of the AF, of thigmotactic-swimming male gametes moving in an almost linear direction, was similar to that of slightly curved free-swimming gametes (not shown). Beat frequencies of free- and thigmotactic-swimming gametes were nearly the same (53-54 Hz), and there was no difference between male and female gametes (Table 1). While, maximum amplitudes of AF, which were measured from the traces of AF waveforms, were $6.3 \pm 0.6 \mu \mathrm{m}(n=5)$ in free-swimming male gametes and $5.2 \pm 0.6 \mu \mathrm{m}(\mathrm{n}=5)$ in thigmotactic-swimming ones

\section{PF movements of free- and thigmotactic-swimming gametes}

PF waveforms of free-swimming gametes showed continuous oscillations, with the deflection angle ranging from $-20.6^{\circ}$ to $29.8^{\circ}$ (Table 1, Figs 14,16 ). While, those of thigmotactic-swimming ones usually showed no motion, or occasional small beats with 
the deflection angle ranging between $22.3^{\circ}$ and $24.0^{\circ}$ (Table 1, Figs 15, 16). A similar tendency was observed in female gametes (Table 1). It was suggested that not only AF waveforms but also PF waveforms were different between free and thigmotactic-swimming gametes.

\section{Chemoorientation of male gametes}

When adding male gametes to settled female gametes, about $50 \%$ of male gametes rapidly showed thigmotactic-swimming pattern. The trajectories of these male gametes were converted from linear paths to middle and small circular paths (Fig. 18). Swimming path curvatures of these male gametes (within $150 \mu \mathrm{m}$ from settled female gametes) were mainly between $0.04-0.2 \mu \mathrm{m}^{-1}(\mathrm{n}=115)$ (Fig. 19). Most of them put their ventral side on the surface of the substratum and swam in clockwise circle. Free-swimming male gametes did not show small circular paths, rather they swam in spirals or undulating lines (Fig. 17). Namely, flagellar behaviours of free-swimming male gametes were not affected by the absence or presence of the sex pheromone from settled female gametes. Swimming velocities of thigmotactic-male gametes during chemotaxis were $78.1 \pm 14.3 \mu \mathrm{m} \mathrm{s}^{-1}(\mathrm{n}=108)$, and significantly decreased compared to the control $(\mathrm{P}<0.001)$. Although, the average swimming velocity of free-swimming male gametes was $199.8 \pm 48.4 \mu \mathrm{m} \mathrm{s}^{-1}(\mathrm{n}=83)$. A significant difference in this value was not observed when compared to the control. The AF beat frequencies of the male gametes were constant at $44-46 \mathrm{~Hz}$ despite different distances from the sex pheromone 
source (within $150 \mu \mathrm{m}$ from settled female gametes). A significant difference could be detected when compared to the control $(53-54 \mathrm{~Hz})(\mathrm{P}<0.001)$.

\section{AF movements of male gametes during chemotaxis}

In order to observe the relationship between swimming path curvature and AF waveform, male gametes $\left(0.04-0.28 \mu \mathrm{m}^{-1}\right.$ of path curvature, $\left.\mathrm{n}=13\right)$ under the sex pheromone were analysed (Figs 20-25, Movie S3). We compared these chemotactic male gametes against thigmotactic gametes without the sex pheromone (from -0.008 to $0.008 \mu \mathrm{m}^{-1}$ of path curvature, $\left.\mathrm{n}=7\right)$. The waveforms in the base region (1.05-1.24) and the middle region (1.19-1.30) of AF showed almost symmetric, but the tip region sometimes became highly asymmetric during chemotaxis resulting in a decrease in R-bend curvature (Fig. 26, S5). Although a significant difference of the AF to the control in the tip region (Fig. 26, $\mathrm{P}<0.05$ ) was observed, a correlation between the path curvature and the AF asymmetric index could not be detected (Fig. 27).

The average value of the AF deflection angle of the chemotactic male gametes was $51.0^{\circ} \pm 23.6^{\circ}$ and that of the thigmotactic-swimming male gametes without the sex pheromone was $-22.9^{\circ} \pm 4.5^{\circ}$ (Table 1 , Fig. 28). There was a significant difference between them $(\mathrm{P}<0.001)$. However, $\mathrm{t}$ here is no correlation between the deflection angle of the AF and the circular path curvature $\left(0.04-0.28 \mu^{-1}\right)$ (Fig. 29). The maximum amplitude of AF was almost the same in chemotactic (5.4 $\pm 0.2 \mu \mathrm{m}, \mathrm{n}=7)$ and thigmotactic (5.2 $\pm 0.6 \mu \mathrm{m}, \mathrm{n}=7)$ swimming gametes. 
PF movements of male gametes during chemotaxis

PF unilateral beating of male gametes was occasionally observed when they were close (less than $100 \mu \mathrm{m}$ ) to the pheromone source (settled female gametes) (Fig. S6). When PF showed strong unilateral beating, the swimming-path curvature of male gametes suddenly increased, namely the swimming direction of male gametes strongly changed. Beat frequency of the PF increased when male gametes were closer to the pheromone source. Several beats of the PF were also detected when they were further away from the pheromone source (Fig. 30). However, the exact timing of PF beating was still not known. Figs 31-64 show two cases of PF beating of male gametes around settled female gametes, 1) PF bent for a while (Figs 31-47) and 2) PF repeated beating (Figs 48-64). Maximum and minimum values of the PF deflection angles became $151.3^{\circ}$ and $12.0^{\circ}\left(0.06-0.20 \mu \mathrm{m}^{-1}\right.$ of path curvatures, $\left.n=4\right)$, (Figs 31-64). The forward stroke of the PF took a longer time (8-16 ms) than the recovery stroke (5-8 ms) (Figs 47, 64, Movie S4). It also became clear that the AF waveform was not affected by the PF waveform in chemotactic male gametes (Figs 47, 64).

\section{Discussion}

Brown algal swarmers (heterokont algae, Stramenopiles) including gametes and zoospores have morphologically and functionally heterogeneous flagella. This is quite different from the cases of the green alga, Chlamydomonas, and the sperm of marine invertebrates. In this study, swimming velocities of E. siliculosus male and female gametes were about $200-240 \mu \mathrm{m} \mathrm{s}^{-1}$ in the free-swimming case and $130-150 \mu \mathrm{m} \mathrm{s}^{-1}$ in 
thigmotactic-swimming one. This value was similar to the previous observation using 16 mm film (Geller \& Müller, 1981). Although the number, length and beat frequency of flagella are diverse, swimming speeds of gametes or sperm in aquatic organisms are not so variable. Some of these values are $174 \mu \mathrm{m} \mathrm{s}^{-1}$ in Chlamydomonas reinhardtii $\mathrm{P}$. A. Dangeard (Goodenough, 1983), $165 \mu \mathrm{m} \mathrm{s}^{-1}$ in sea urchin (Wood et al., 2005), 276 $\mu \mathrm{m} \mathrm{s}^{-1}$ in ascidians (Yoshida et al., 2002), and $375 \mu \mathrm{m} \mathrm{s}^{-1}$ in starfish (Shiba et al., 2006).

Thigmotactic-swimming gametes of E. siliculosus did not show helical rotations of their cell body and they displayed only two-dimensional waveforms of the AF. While, free-swimming gametes frequently showed helical rotations of their cell body and they displayed two- and three-dimensional waveforms of the AF. It was mathematically shown by Chwang \& Wu (1971) and Keller \& Rubinow (1976) that three-dimensional (helical) flagellar movement induces rotation of the cell. Helical rotations of Ectocarpus gametes would be induced by a combination of several complex AF waveforms as the suggestions of Geller and Müller (1981). Although there is no significant difference in beat frequency of the AF between free- and thigmotactic-swimming gametes, swimming velocities between them were different. AF amplitude also did not seem to be effective to the gamete swimming speed. An increase in swimming speed in free-swimming gametes may be caused by helical rotation of the gamete cell body. While, Ishijima (2012) examined the movement characteristics of the sperm and flagella from a lancelet and 35 species of fishes using high-speed video microscopy. He concluded that flagellar beat frequency was most effective to the swimming speed, neither the flagellar wavelength nor amplitude. 
After Müller $(1967,1971)$ first discovered the brown algal pheromone 'ectocarpene' in Ectocarpus siliculosus, many other sex pheromones in brown algae were identified (see the reviews of Maier \& Müller, 1986 and Maier, 1993). As a result of these studies, detailed observations of the behaviour of male and female gametes during the fertilization process of E. siliculosus were carried out. Ectocarpus male and female gametes are morphologically isomorphic. Female gametes settle down on the substratum quicker than male gametes. When settled, the female gametes begin to secret the sex pheromone. However, the locomotion and flagellar waveforms of male and female gametes were observed to be almost same after adding them into the chamber.

During chemotaxis in the presence of sex pheromone, the AF deflection angle of male gametes significantly changed from the control with decreasing beat frequency of the AF. Geller \& Müller (1981) showed a correlation between the average deflection angle of the AF and the radius of the track. In addition to their results, we clarified that the deflection angle of the AF was significantly changed when male gametes altered their swimming pattern from highly linear paths to small or medium circular paths. However, the deflection angle did not significantly change when the gametes switched from medium to small circular paths. Therefore, it was suggested that changing the swimming paths of male gametes from a highly linear path to a circular path was affected by the AF itself. While, alternating the trajectory from a medium circular to a small circular path was attributed to the PF.

Geller \& Müller (1981) and Maier \& Calenberg (1994) showed that the PF performed fast and unilateral beats, when the cell sensed decreasing concentration of 
pheromone. Unilateral beats of the PF were usually observed for reorientation of male gametes towards the sex pheromone source (settled female gametes). In this study, it became clear that, regardless of unilateral beating of the PF, the waveform of the AF remained stable (Figs 47, 64). It was suggested that the AF and PF would move independently in the presence of sex pheromone.

Matsunaga et al. (2010) showed the rapid lateral beats of the PF during phototactic-orientation of gametes in the brown alga, Scytosiphon lomentaria. Those studies reveal that the forward stroke of PF took a longer time (6-8 ms) than the recovery stroke (2-4 ms). When the gametes show phototactic turning, AF undulation ceased. Therefore, AF motion during phototactic turning of gametes is clearly different from the case of the chemotactic one, although unilateral beat patterns of the PF seem to be similar.

It was revealed that the AF plays a role in changing gamete locomotion from freeto thigmotactic-swimming and also from straight to circular swimming. Moreover, the AF changes the flagellar asymmetry and deflection angle during these steps but it does not change these properties in the presence of the sex pheromone. The PF shows irregular changes in the deflection angle and large bending are dependent on the concentration gradient of the sex pheromone. The exact timing and mechanism of large unilateral beats in the PF during chemotaxis remain unclear. In the ascidian, Yoshida \& Yoshida (2011) report that sperm attractants appear to induce $\mathrm{Ca}^{2+}$ entry from extracellular spaces into sperm cells. This increases the intracellular $\mathrm{Ca}^{2+}$ concentration which mediates the beating patterns of sperm flagella. This increase in $\mathrm{Ca}^{2+}$ 
concentration results in the observed chemotactic turn. Maier and Calenberg (1994) report on the effects of calcium on flagellar movements of E. siliculosus male gametes during chemotaxis. They surveyed several calcium antagonists and channel blockers including Lanthanum, Ruthenium Red, Verapamil, Nifedipine, and Trifluoperazine, and showed that: 1) the asymmetric bending of the AF of male gametes will be strongly inhibited by $\left.\mathrm{La}^{3+}, 2\right)$ Nefedipine might interact with $\mathrm{Ca}^{2+}$ channels which are involved in the action of PF, and 3) Trifluoperazine might bind to and activate the pheromone receptors in Ectocarpus via hydrophobic interactions. Recently, Fu et al. (2014) conducted a proteomics analysis of flagella of the brown alga, Colpomenia bullosa (Saunders) Yamada suggested that $8 \%$ of the 495 flagellar proteins were related to proteins with calcium-binding function. Further investigation on the roles of calcium in flagellar bending using a high-speed camera will clarify the characteristic beat patterns of the heterogeneous AF and PF of brown algal gametes during chemotaxis.

\section{Acknowledgement}

This study was partially supported by the Japanese Association for Marine Biology (25-55, 26-56). 


\section{Reference}

Baba, S.A. \& Mogami, Y. (1985). An approach to digital image analysis of bending shapes of eukaryotic flagella and cilia. Cell Motility, 5: 475-489.

Bouck, G.B. (1969). Extracellular microtubules: the origin, structure, and attachment of flagellar hairs in Fucus and Ascophyllum antherozoids. Journal of Cell Biology, 40: 446-460.

Andersen, R.A. (2004). Biology and systematics of heterokont and haptophyte algae. American Journal of Botany, 91: 1508-1522.

Chwang, A.T. \& Wu, T.Y. (1971). A note on the helical movement of micro-organisms. Proceedings of the Royal Society of London. Series B, Biological Sciences, 178: $327-346$.

Clayton, M. N. (1989). Brown algae and chromophyte phylogeny: In The Chromophyte Algae: problems and perspectives. (Green, J.C., Leadbeater, B.S.C.\& Diver, W.L. editors), The Systematics Association Special, 38: 230-253. Clarendon Press, Oxford, UK.

Geller, A. \& Müller, D.G. (1981). Analysis of the flagellar beat pattern of male Ectocarpus siliculosus gametes (Phaeophyta) in relation to chemotactic stimulation by female cells. Journal of Experimental Biology, 92: 53-66.

Gibbons, I.R. (1981). Cilia and flagella of eukaryotes. Journal of Cell Biology, 91(suppl): 107s-124s.

Goodenough, U.W. (1983). Motile detergent-extracted cells of Tetrahymena and Chlamydomonas. Journal of Cell Biology, 96: 1610-1621. 
Holwill, M.E.J. \& Sleigh, M.A. (1967). Propulsion by hispid flagella. Journal of Experimental Biology, 47: 267-276.

Inaba, K. (2003). Molecular architecture of the sperm flagella: molecules for motility and signaling. Zoological Science, 20: 1043-1056.

Ishijima, S. (2012). Comparative analysis of movement characteristics of lancelet and fish spermatozoa having different morphologies. The Biological Bulletin, 222: 214-221.

Jahn, T.L., Landman, M.D. \& Fonseca, J.R. (1964). The mechanism of locomotion of flagellates. II. Function of the mastigonemes of Ochromonas. Journal of Protozoology, 11: 291-296.

Kaupp, U.B., Kashikar, N.D. \& Weyand, I. (2008). Mechanisms of sperm chemotaxis. Annual Reviews Physiology, 70: 93-117.

Kawai, H., Müller, D.G., Folster, E. \& Häder, D.P. (1990). Phototactic responses in the gametes of the brown alga, Ectocarpus siliculosus. Planta, 182: 292-297

Kawai, H. \& Kreimer, G. (2000). Sensory mechanisms: Light perception and taxis in algae. In The flagellates: Unity, Diversity and Evolution (Leadbeater, B. \& Green, J., editors), 124-146. Taylor and Francis, London and New York.

Keller, J.B. \& Rubinow, S.I. (1976). Swimming of flagellated microorganisms. Biophysical Journal, 16: 151-170.

Maier, I. (1993). Gamete orientation and induction of gametogenesis by pheromone in algae and plants. Plant, Cell \& Environment, 16: 891-907. 
Maier, I. (1995). Brown algal pheromones. In Progress in Phycological Research (Round, F. E. \& Chapman, D. J., editors), 11: 51-102. Biopress, Bristol, UK.

Maier, I., \& Müller, D.G. (1986). Sexual pheromones in algae. Biological Bulletin, 170: 145-175.

Maier, I. \& Calenberg, M. (1994). Effect of extracellular $\mathrm{Ca}^{2+}$ and $\mathrm{Ca}^{2+}$ antagonists on the movement and chemoorientation of male gametes of Ectocarpus siliculosus (Phaeophyceae). Botanica Acta, 107: 451-460.

Matsunaga, S., Uchida, H., Iseki, M., Watanabe, M. \& Murakami, A. (2010). Flagellar motions in phototactic steering in a brown algal swarmer. Photochemistry and Photobiology, 86: 374-381.

Miller, R.L. (1975). Chemotaxis of the spermatozoa of Ciona intestinalis. Nature, 254: 244-245.

Miller, R.L. (1977). Chemotactic behavior of the sperm of chitons (Mollusca: Polyplacophora). Journal of Experimental Zoology, 202: 203-211.

Miller, R. L. \& Brokaw, C. J. (1970). Chemotactic turning behaviour of Tubularia spermatozoa. Journal of Experimental Biology, 52: 699-706.

Mitchell, D.R. (2000). Chlamydomonas flagella. Journal of Phycology, 36: 261-273.

Müller. D.G. (1967) Ein leicht flüchtiges Gyno-Gamon der Braunalge Ectocarpus siliculosus. Naturwissenschaften, 54: 496-497.

Müller, D.G. (1978). Locomotive responses of male gametes to the species-specific sex attractant in Ectocarpus siliculosus (Phaeophyta). Archiv für Protistenkunde, 120: 371-377. 
Müller, D.G. \& Falk, H. (1973). Flagellar structure of the gametes of Ectocarpus siliculosus (Phaeophyta) as revealed by negative staining. Archiv für Mikrobiologie, 91: 313-322.

Müller, D.G., Jaenicke, L., Donike, M. \& Akintobi, T. (1971). Sex attractant in a brown alga: chemical structure. Science, 171: 815-817.

O' Kelly, C.J. (1989). The evolutionary origin of the brown algae: Information from studies of motile cell structure. In The Chromophyte Algae: problems and perspectives. (Green, J.C., Leadbeater, B.S.C.\& Diver, W.L. editors), The Systematics Association Special, 38: 256-278. Clarendon Press, Oxford, UK.

Provasoli, L. (1968). Media and prospects for the cultivation of marine algae. In Cultures and collections of algae (Watanabe, A. \& Hattori, A., editors), 63-75. Japanese Society of Plant Physiology, Hakone.

Shiba, K., Tagata, T., Ohmuro, J., Mogami, Y., Matsumoto, M., Hoshi, M. \& Baba, S. A. (2006). Peptide-induced hyperactivation-like vigorous flagellar movement in starfish sperm. Zygote, 14: 23-32.

Shiba, K., Baba, S. A., Inoue, T., \& Yoshida, M. (2008). $\mathrm{Ca}^{2+}$ bursts occur around a local minimal concentration of attractant and trigger sperm chemotactic response. Proceedings of the National Academy of Sciences of the United States of America, 105: 19312-19317.

Wood, C.D., Nishigaki, T., Furuta, T,, Baba, S. A., Darszon, A. (2005) Real-time analysis of the role of $\mathrm{Ca}^{2+}$ in flagellar movement and motility in single sea urchin sperm. Journal of Cell Biology, 169: 725-731. 
Yoshida, M., Murata, M., Inaba, K., \& Morisawa, M. (2002). A chemoattractant for ascidian spermatozoa is a sulfated steroid. Proceedings of the National Academy of Sciences of the United States of America, 99: 14831-14836.

Yoshida, M. \& Yoshida, K. (2011). Sperm chemotaxis and regulation of flagellar movement by $\mathrm{Ca}^{2+}$. Molecular Human Reproduction, 17: 457-465. 


\section{Figure legends}

Figs 1-3. The trajectories of free- and thigmotactic-swimming male gametes in $E$. siliculosus. 1, 2. The swimming trajectories of free-swimming male gametes (1) and thigmotactic-swimming male gametes (2) are obtained by image processing during a period of 2s. 3. Frequency of the swimming path curvatures (absolute value) of thigmotactic-swimming male gametes $(\mathrm{n}=115)$. Scale bars $=100 \mu \mathrm{m}$.

Figs 4-13. The anterior flagellar (AF) waveforms of free- and thigmotactic-swimming male gametes. 4-6. A typical flagellar waveform of free-swimming gametes (symmetrical waveform of the AF) recorded at 1.6-ms intervals. 7-9. A typical flagellar waveform of thigmotactic-swimming male gametes recorded at 1.6-ms intervals. 10, 11 . Trace of AF waveforms in free-swimming male gametes (10) and thigmotactic-male gametes (11). Twenty frames were chosen every $1.6 \mathrm{~ms}$ and traces of the AF were merged. Black bars show $5 \mu \mathrm{m}$ reference line and grey dots indicate the anterior end of the cell body. 12. Flagellar asymmetric indices of free- and thigmotactic- swimming male gametes. I. Basal region of the AF; II. Middle region; III. Tip region; IV. Whole region. Free- vs. thigmotactic-swimming male gametes, $* \mathrm{P}<0.001$. 13. Average value of the deflection angle at $8 \mu \mathrm{m}$ from the tip of the AF. I. Free-swimming male gametes; II. Thigmotactic-male gametes. Free- vs. thigmotactic-swimming male gametes, $* \mathrm{P}<0.001$. Scale bars $=5 \mu \mathrm{m}$.

Figs 14-16. The posterior flagellar (PF) waveforms of free- and thigmotactic-swimming male gametes. 14, 15. Trace of the PF waveforms in free- (14) and thigmotactic-swimming male gametes (15). Twenty forms were chosen every $1.6 \mathrm{~ms}$ 
and traces of the PF were merged. Black bars show $5 \mu \mathrm{m}$ reference line and gray dots indicate the anterior end of the cell body. 16. The deflection angle at $1 \mu \mathrm{m}$ from the tip of PF is plotted against time.

Figs 17-19. The trajectories of chemotactic male gametes. 17, 18. The swimming trajectories of chemotactic gametes are obtained by image processing during the period of 2s. 17. Free-swimming male gametes on the upper region from settled female gametes. 18. Thigmotactic-swimming male gametes near settled female gametes. White arrowheads shows settled female gametes. White double arrowheads shows the swimming path of 20. 19. Frequency of the swimming path curvatures $(n=115)$. Scale bars $=100 \mu \mathrm{m}$.

Figs 20-29. The AF beat patterns of chemotactic male gametes. 20-25. Trajectory (20) and typical flagellar waveforms were recorded at 1.6-ms intervals (21-25). 26. The AF asymmetric indices of chemotactic male gametes. I. Base region; II. Middle region; III. Tip region; IV. Whole region. Control vs. Chemotactic gametes, ${ }^{* *} \mathrm{P}<0.05 .27$. Asymmetric indices of tip region in the AF plotted against path curvatures. 28. Average value of deflection angle at $8 \mu \mathrm{m}$ from the tip of AF. I. Control; II. Chemotactic gametes. Control vs. chemotactic gametes, ${ }^{*} \mathrm{P}<0.001$. 29. Average value of deflection angles at $8 \mu \mathrm{m}$ from the tip of AF plotted against path curvatures. Scale bars $=10 \mu \mathrm{m}$. Fig. 30. A trajectory and PF bending pattern of a chemotactic male gamete $\left({ }^{\top}\right)$ near female gamete (우). White squares show the timing of lateral PF beating of male gamete. Gray squares show the motionless PF. A-F show detailed images of male 
gametes at each square, respectively. Arrows indicate the position of the PF. Scale bar = $20 \mu \mathrm{m}$ in 30, and $10 \mu \mathrm{m}$ in 30A-F.

Figs 31-64. Two patterns of lateral beats of the PF in chemotactic male gametes. PF bent for a while (31-47) and PF repeated beating (48-64). Images were recorded at 1.6-ms intervals under the same conditions (45-47 were analysed from 31-44, and 62-64 were analysed from 48-61). 45, 62. Trajectories of chemotactic male gametes. Arrows indicate the timing of unilateral beats of PF. 46, 63. Trace of AF and PF waveforms. Twenty forms were chosen every $1.6 \mathrm{~ms}$ and merged. Black bars show $5 \mu \mathrm{m}$ reference line and grey dots indicate the anterior end of cell body. 47, 64. Deflection angles at 8 $\mu \mathrm{m}$ from the tip of the AF and at $1 \mu \mathrm{m}$ from the tip of the PF are plotted. Arrows indicate the timing of unilateral beats of PF in $\mathbf{4 5}$ and $\mathbf{6 2}$. Scale bars $=10 \mu \mathrm{m}$. Figs S1, 2. Definitions of the terms. 1. Definition of the swimming path curvature $(\Gamma)$ and the flagellar curvature $(\gamma)$. Blue dots and lines show the center of cell body and zigzag trajectory of the cell body. 2. Definition of the deflection angle $(\theta)$.

Figs S3-5. AF curvature plotted against distance from near the base of the flagellum. The basal part of AF was hidden by the cell body, therefore the distance of the base of AF was obtained by adding 4 um (as the half width of the cell body) to the AF length AF which was detected under microscopy. Data from 20 waveforms were merged. 3. Free-swimming male gametes. 4. Thigmotactic-swimming male gametes. 5. Chemotactic-male gametes.

Fig. S6. Distribution of deflection angles of the PF of the control $(n=148$ plots from 5 male gametes) and chemotactic-male gametes ( $n=148$ plots from 4 male gametes). 
Movie S1. Free-swimming male gametes.

Movie S2. Thigmotactic-swimming male gametes.

Movie S3. Chemotactic-male gametes.

Movie S4. Rapid lateral beats of the PF of male gametes. 


\section{Flagellar Waveforms of gametes in the brown alga Ectocarpus siliculosus}

Nana Kinoshita ${ }^{1}$, Kogiku Shiba ${ }^{2}$, Kazuo Inaba ${ }^{2}$, Gang Fu ${ }^{3}$, Chikako Nagasato ${ }^{3}$ and Taizo Motomura $3^{3 *}$

${ }^{1}$ Graduate School of Environmental Science, Hokkaido University, Sapporo 060-0810, Hokkaido, Japan

${ }^{2}$ Shimoda Marine Research Center, University of Tsukuba, Shimoda 415-0025, Shizuoka, Japan

${ }^{3}$ Muroran Marine Station, Field Science Center for Northern Biosphere, Hokkaido University, Muroran 051-0003, Hokkaido, Japan

*Corresponding author: Taizo Motomura

Email: motomura@fsc.hokudai.ac.jp

Tel: +81 143222846

Fax: +81 143224135

A running title: Heterogeneous flagella in brown algae 


\section{Abstract}

Brown algae are members of the Stramenopiles and their gametes generally have two heterogenous flagella: a long anterior flagellum (AF) with mastigonemes, and a short posterior flagellum (PF). In this study, swimming paths and flagellar waveforms in free-swimming and thigmotactic-swimming male and female gametes, and male gametes during chemotaxis, were quantitatively analysed in the model brown alga Ectocarpus siliculosus. This was performed using a high-speed video camera. It was revealed that AF plays a role in changing the locomotion of male and female gametes from free-swimming to thigmotactic-swimming, and also in changing the swimming path of male gametes from linear to circular during chemotaxis. In the presence of a sex pheromone, male gametes changed their swimming path from linear (swimming path curvature, $0-0.02 \mu \mathrm{m}^{-1}$ ) to middle and small circular path (swimming path curvature, $0.04-0.20 \mu \mathrm{m}^{-1}$ ). The flagellar asymmetry and the deflection angle of the AF became larger, whereas the oscillation pattern of the AF was stable. However, there was no correlation between those of the AF and the path curvature when the male gametes showed middle to small circular paths. PF irregularly changed the deflection angle, and the oscillation pattern was unstable depending on the gradient of the sex pheromone concentration. AF waveforms were independent of PF locomotion during chemotaxis. It is quantitatively suggested that the AF has the ability to change the swimming path of male gametes-for example, from a highly linear path to a circular path-while changes in locomotion from a middle circle path to a small circle path is the result of beating of the PF. 
Key words: anterior flagellum, brown algae, chemotaxis, flagellar movement, gametes, posterior flagellum, thigomotaxis 


\section{Introduction}

Motile gametes of the brown algae usually have two heterogeneous flagella, a long anterior flagellum (AF) and a short posterior flagellum (PF) (Clayton, 1989; O' Kelly, 1989; Anderson, 2004). The AF is decorated with mastigonemes and generates a force for moving forward of gametes (Jahn et al., 1964; Holwill \& Sleigh, 1967; Bouck, 1969). The PF frequently has a basal swelling part (paraflagellar body), and provides rapid lateral beats for changing the direction of the cell movement (Kawai \& Kreimer, 2000; Maier, 1995).

Chemotactic responses of male gametes to a sex pheromone from settled female gametes have been studied for many years in the isogamous brown alga, Ectocarpus siliculosus (Dillwyn) Lyngbye (Müller \& Falk, 1973; Müller, 1978; Geller \& Müller, 1981). First, female gametes shortly settle on the substratum and secrete the sex pheromone ‘ectocarpen’ (Müller, 1967, Müller et al., 1971). Second, free-swimming male gametes began to swim in close contact with the surface of slide glass or cover slip. This response is enhanced by the presence of the sex pheromone from the female gamete, and is regarded as thigmotaxis (Müller, 1978; Geller \& Müller, 1981). The swimming velocity of male gametes decreased, and the swimming radius became smaller. Third, the male gamete changes the AF waveform, which causes the cell body to move in circular paths under existence of the sex pheromone. This behaviour of the male gamete is regarded as chemokinesis (Maier, 1993, 1995). PF of the male gamete performs fast, unilateral beating, when it detects a decrease of the sex pheromone concentration gradients. This PF behaviour results in a reorientation towards the 
pheromone source and is defined as chemoklinotaxis (Maier, 1993, 1995). Afterwards, the male gamete anchors itself with the tip of the AF on the surface of the female gamete, and finally, the male gamete fuses to the female one (Müller \& Falk, 1973). Therefore, the AF and PF of male gametes have crucial roles in approach and contact with female gametes.

Geller \& Müller (1981) firstly reported that the AF of male gametes in $E$. siliculosus perform asymmetric bending in the presence of the sex pheromone, which shows a negative linear correlation between the average of deflection angle of the AF from the cell axis and the radius of the track of the male gametes during chemokinesis. Their analysis was based on measuring the deflection of the first visible bend of the AF from the cell axis. However, information on the flagellar waveform of brown algae has been limited compared to the case of metazoa (Gibbons, 1981; Inaba, 2003) and the green alga, Chlamydomonas (Mitchell, 2000). Analyses of flagellar waveforms in sperm of marine invertebrates using high-speed camera revealed that flagellar waveforms became asymmetric when sperm changed the swimming direction toward the sex pheromone source. Their flagellar waveforms remained symmetric when sperm swam directly toward the pheromone source (Miller \& Brokaw, 1970; Miller, 1975; Miller, 1977). It has been reported that $\mathrm{Ca}^{2+}$ was a primary factor regulating the symmetry and asymmetry of flagellar waveforms (Kaupp et al., 2008; Yoshida \& Yoshida, 2011).

In the present study, swimming paths and two heterogeneous flagellar waveforms of male and female gametes of the brown alga E. siliculosus were quantitatively analysed using a high-speed camera, focusing on free- and thigmotactic-swimming male 
and female gametes and chemotactic-swimming male gametes to understand the more precise relationship between $\mathrm{AF}$ and $\mathrm{PF}$ waveforms of male gametes during fertilization in brown algae.

\section{Materials and methods}

Preparation of gametes

Male and female gametophytes (strain Ec32m and Ec25f) of Ectocarpus siliculosus (Dillw.) Lyngbye were gifts from Drs Peters, A. F. (Bezhin Rosko, France) and Coelho, S. M. (UMR 7139 CNRS-UPMC, Station Biologique de Roscoff, France). These gametophytes were cultured in the half strength PES medium (Provasoli, 1968) under cool white fluorescent lamps $\left(30-40 \mu \mathrm{mol} \mathrm{m}^{-2} \mathrm{~s}^{-1}\right)$ at $15{ }^{\circ} \mathrm{C}$ in long day conditions $(14 \mathrm{hr}$ light / 10 hr dark). Two days after changing to new medium, gametes were released from plurilocular gametangia within $2 \mathrm{hr}$ of light irradiation. They showed a strong negative phototaxis and gathered at the opposite side of culture dishes from the light source.

Observations and recording

Thirty $\mu \mathrm{l}$ of culture medium containing freshly liberated male and female gametes was added in an observation chamber (90 $\mu$ m depth). This observation chamber was made of double-faced adhesive tape between a slide glass and a cover slip. Images of flagellar waveforms were recorded with a phase contrast microscope (BX51, Olympus, Tokyo, Japan) with a 40× objective (UPlan FLN, Olympus) connected to a high-speed CCD 
camera (HAS220, Ditect, Tokyo, Japan) at 200 or 600 fps (frames per sec) and 1/1000 sec shutter speed. Observations were carried out using an R-58 red cut-off glass filter (S-058, HOYA, Tokyo, Japan) in the dark room at room temperature (ca. $20^{\circ} \mathrm{C}$ ), for preventing the effect of blue light on phototaxis of E. siliculosus (Kawai et al., 1990). Image analyses of flagellar waveforms were executed only on planner bending waveforms.

\section{Data analysis}

Swimming velocities, swimming path curvatures, flagellar curvatures and the deflection angle of gametes were analysed by Bohboh software (Bohboh Soft, Tokyo, Japan). Images of the cell body of gametes were automatically tracked, and the swimming velocities were measured along the zigzag trajectory of the cell bodies during flagellar beating cycles (trajectory swimming velocity). The path curvature, which is the reciprocal of the radius of curvature $(\rho)$ of the circular swimming path, was also calculated (Fig. S1). Trajectories in a clockwise direction were defined as a plus and trajectories in a counterclockwise direction were defined as a minus. The flagellar waveforms of gametes were automatically traced. The flagellar curvature, which is the reciprocal of the radius of curvature (r) of the flagellar waveform, was calculated based on the method of Baba and Mogami (1985). Flagellar asymmetry was expressed as the ratio of the maximal curvature of $\mathrm{P}$ bend (Principal bend) and that of $\mathrm{R}$ bend (Reverse bend) ( $\mathrm{P}$ bend Max / $\mathrm{R}$ bend Max). The ratio should be equal to one when the flagella show completely symmetric bending, and is should be greater than one when they show 
an asymmetric waveform. This ratio is referred to as the asymmetric index (Shiba et al., 2008).

The angle of the tangent to the flagellar shaft was measured with respect to the direction of the axis of the cell body. It was referred to the deflection angle (Fig. S2). The right side was defined as plus and the left side was defined as minus, when the AF was localized to the direction of forward movement and viewed from the ventral side of the gametes. The anterior end of cell body was defined as zero with respect to the deflection angle of the AF and the posterior end of cell body was defined as zero in with respect to the deflection angle of the PF. The points which tended to take on large values in maximum and minimum of the deflection angle was $8 \mu \mathrm{m}$ from the tip of the AF and $1 \mu \mathrm{m}$ from the tip of the PF.

\section{Results}

Locomotion of free- and thigmotactic-swimming gametes

Two patterns of swimming were observed in male and female gametes of E. siliculosus. The first observed pattern was that the gametes freely swam with helical rotations of the cell body without touching anything. Trajectories of these gametes showed spiral or undulating lines (Fig. 1). The second observed pattern was that they swam just on the substratum by eliminating the helical rotation of the cell bodies. These thigmotactic-swimming male and female gametes followed straight to large circular paths (Fig. 2). 
Just after adding $30 \mu \mathrm{l}$ of culture medium containing freshly liberated male or female gametes to a chamber, about $20 \%$ of gametes showed thigmotaxis and $80 \%$ of gametes freely swam. After three min, about $50 \%$ of gametes showed thigmotactic-swimming. The number of thigmotactic-swimming gametes gradually increased.

The swimming velocity was $205.2 \pm 49.5 \mu \mathrm{m} \mathrm{s}^{-1}(\mathrm{n}=95)$ in free-swimming male gametes and $131.5 \pm 24.1 \mu \mathrm{m} \mathrm{s}^{-1}(\mathrm{n}=115)$ in thigmotactic-swimming gametes, and there were a significant difference $(\mathrm{P}<0.001)$ (Table 1) between the two values. In female gametes, the swimming velocity was $191.0 \pm 48.4 \mu \mathrm{m} \mathrm{s}^{-1}(\mathrm{n}=90)$ in free-swimming gametes and $151.8 \pm 38.6 \mu \mathrm{m} \mathrm{s}^{-1}(\mathrm{n}=115)$ in thigmotactic-swimming gametes. There was also a significant difference $(\mathrm{P}<0.001)$ between these values. No significant difference was observed between the swimming patterns of male and female gametes.

The path curvature, which is the reciprocal number of the radius of curvature $(\rho)$ of the circular-swimming gametes, was calculated. Trajectories in a clockwise rotation were defined as plus and those in a counterclockwise direction were defined as minus (Fig. S1). Thigmotactic-swimming male gametes showed highly linear to large circular paths without a pheromone source, and $66 \%$ of the path curvature values ranged from $-0.02-0.02 \mu m^{-1}(n=115)$ (Fig. 3). Similar tendencies were also observed in female gametes.

AF movements of free- and thigmotactic-swimming gametes 
Flagellar waveform of free- and thigmotactic-swimming male and female gametes, which showed typical swimming velocities and path curvatures, were quantitatively analysed. The AF showed periodic oscillations, but the PF showed only irregular oscillations in Ectocarpus gametes. Therefore, flagellar curvature, asymmetric index and beat frequency were analysed only in the AF.

AF movements of free-swimming gametes were composed of symmetric and asymmetric waveforms (Figs 4-6, 10, Movie S1). The AF of thigmotactic-swimming gametes usually showed asymmetric waveforms (Figs 7-9, 11, Movie S2). In the case of free-swimming male gametes, which showed the highly linear paths (path curvature $\left.-0.01-0.009 \mathrm{~m}^{-1}\right)(\mathrm{n}=5)$, maximum values of P-bend and R-bend in the AF were 1.13 \pm 0.09 and $1.08 \pm 0.06$, respectively. The asymmetric index ( $\mathrm{P}$ bend / $\mathrm{R}$ bend) of the AF was $1.05 \pm 0.03$ as the whole region (Fig. 12, S3). The slightly curved free-swimming male gametes $\left(>0.012 \mu \mathrm{m}^{-1}\right)$ had a asymmetric waveform which was similar to those of thigmotactic-swimming gametes (not shown). The AF of thigmotactic-swimming male gametes, which showed highly linear paths (from -0.008 to $0.008 \mu \mathrm{m}^{-1}, \mathrm{n}=7$ ), displayed asymmetric waveforms (Fig. 12). The maximum values of P-bend and R-bend were $1.16 \pm 0.13$ and $0.98 \pm 0.12$, respectively (Fig. S4). Next, we separately measured the asymmetric index at the base, middle and tip regions of AF (Fig. 12) for examining which part of AF showed a conspicuous asymmetry. The asymmetric indices were $1.05 \pm 0.07$ at the base $(0-5 \mu \mathrm{m}), 1.19 \pm 0.045$ at the middle $(6-10 \mu \mathrm{m}), 1.42 \pm$ 0.15 at the tip $(11-14 \mu \mathrm{m})$, and $1.24 \pm 0.075$ as the whole region $(0-14 \mu \mathrm{m})$. The AF waveform of these two types of gametes, which showed highly linear paths, were 
significantly different in asymmetric index, especially between the tip region and the whole region $(\mathrm{P}<0.001)$ (Fig. 12). Female gametes also showed similar patterns of AF waveforms. The asymmetric waveform in AF was observed in both free- and thigmotactic-swimming gametes; however, the symmetric waveform in the AF was observed only in free-swimming gametes during straight swimming.

The deflection angle between the flagellum and the axis of cell body was obtained. The average values of the deflection angle in the AF of free- and thigmotactic-swimming male gametes, which showed a highly linear path, were $6.3^{\circ} \pm$ $8.0^{\circ}\left(\max 102.6^{\circ}\right.$, $\left.\min 80.8^{\circ}\right)$ and $-22.9^{\circ} \pm 4.5^{\circ}\left(\max 45.2^{\circ}\right.$, min $\left.-106.6^{\circ}\right)$, respectively (Table 1, Fig. 13). There was a statistically significant difference between these two values ( $\mathrm{P}<0.001)$. The deflection angle of the AF, of thigmotactic-swimming male gametes moving in an almost linear direction, was similar to that of slightly curved free-swimming gametes (not shown). Beat frequencies of free- and thigmotactic-swimming gametes were nearly the same (53-54 Hz), and there was no difference between male and female gametes (Table 1). While, maximum amplitudes of AF, which were measured from the traces of AF waveforms, were $6.3 \pm 0.6 \mu \mathrm{m}(n=5)$ in free-swimming male gametes and $5.2 \pm 0.6 \mu \mathrm{m}(\mathrm{n}=5)$ in thigmotactic-swimming ones

\section{PF movements of free- and thigmotactic-swimming gametes}

PF waveforms of free-swimming gametes showed continuous oscillations, with the deflection angle ranging from $-20.6^{\circ}$ to $29.8^{\circ}$ (Table 1, Figs 14,16 ). While, those of thigmotactic-swimming ones usually showed no motion, or occasional small beats with 
the deflection angle ranging between $22.3^{\circ}$ and $24.0^{\circ}$ (Table 1, Figs 15, 16). A similar tendency was observed in female gametes (Table 1). It was suggested that not only AF waveforms but also PF waveforms were different between free and thigmotactic-swimming gametes.

\section{Chemoorientation of male gametes}

When adding male gametes to settled female gametes, about $50 \%$ of male gametes rapidly showed thigmotactic-swimming pattern. The trajectories of these male gametes were converted from linear paths to middle and small circular paths (Fig. 18). Swimming path curvatures of these male gametes (within $150 \mu \mathrm{m}$ from settled female gametes) were mainly between $0.04-0.2 \mu \mathrm{m}^{-1}(\mathrm{n}=115)$ (Fig. 19). Most of them put their ventral side on the surface of the substratum and swam in clockwise circle. Free-swimming male gametes did not show small circular paths, rather they swam in spirals or undulating lines (Fig. 17). Namely, flagellar behaviours of free-swimming male gametes were not affected by the absence or presence of the sex pheromone from settled female gametes. Swimming velocities of thigmotactic-male gametes during chemotaxis were $78.1 \pm 14.3 \mu \mathrm{m} \mathrm{s}^{-1}(\mathrm{n}=108)$, and significantly decreased compared to the control $(\mathrm{P}<0.001)$. Although, the average swimming velocity of free-swimming male gametes was $199.8 \pm 48.4 \mu \mathrm{m} \mathrm{s}^{-1}(\mathrm{n}=83)$. A significant difference in this value was not observed when compared to the control. The AF beat frequencies of the male gametes were constant at $44-46 \mathrm{~Hz}$ despite different distances from the sex pheromone 
source (within $150 \mu \mathrm{m}$ from settled female gametes). A significant difference could be detected when compared to the control $(53-54 \mathrm{~Hz})(\mathrm{P}<0.001)$.

\section{AF movements of male gametes during chemotaxis}

In order to observe the relationship between swimming path curvature and AF waveform, male gametes $\left(0.04-0.28 \mu \mathrm{m}^{-1}\right.$ of path curvature, $\left.\mathrm{n}=13\right)$ under the sex pheromone were analysed (Figs 20-25, Movie S3). We compared these chemotactic male gametes against thigmotactic gametes without the sex pheromone (from -0.008 to $0.008 \mu \mathrm{m}^{-1}$ of path curvature, $\left.\mathrm{n}=7\right)$. The waveforms in the base region (1.05-1.24) and the middle region (1.19-1.30) of AF showed almost symmetric, but the tip region sometimes became highly asymmetric during chemotaxis resulting in a decrease in R-bend curvature (Fig. 26, S5). Although a significant difference of the AF to the control in the tip region (Fig. 26, $\mathrm{P}<0.05$ ) was observed, a correlation between the path curvature and the AF asymmetric index could not be detected (Fig. 27).

The average value of the AF deflection angle of the chemotactic male gametes was $51.0^{\circ} \pm 23.6^{\circ}$ and that of the thigmotactic-swimming male gametes without the sex pheromone was $-22.9^{\circ} \pm 4.5^{\circ}$ (Table 1 , Fig. 28). There was a significant difference between them $(\mathrm{P}<0.001)$. However, $\mathrm{t}$ here is no correlation between the deflection angle of the AF and the circular path curvature $\left(0.04-0.28 \mu^{-1}\right)$ (Fig. 29). The maximum amplitude of AF was almost the same in chemotactic (5.4 $\pm 0.2 \mu \mathrm{m}, \mathrm{n}=7)$ and thigmotactic (5.2 $\pm 0.6 \mu \mathrm{m}, \mathrm{n}=7)$ swimming gametes. 
PF movements of male gametes during chemotaxis

PF unilateral beating of male gametes was occasionally observed when they were close (less than $100 \mu \mathrm{m}$ ) to the pheromone source (settled female gametes) (Fig. S6). When PF showed strong unilateral beating, the swimming-path curvature of male gametes suddenly increased, namely the swimming direction of male gametes strongly changed. Beat frequency of the PF increased when male gametes were closer to the pheromone source. Several beats of the PF were also detected when they were further away from the pheromone source (Fig. 30). However, the exact timing of PF beating was still not known. Figs 31-64 show two cases of PF beating of male gametes around settled female gametes, 1) PF bent for a while (Figs 31-47) and 2) PF repeated beating (Figs 48-64). Maximum and minimum values of the PF deflection angles became $151.3^{\circ}$ and $12.0^{\circ}\left(0.06-0.20 \mu \mathrm{m}^{-1}\right.$ of path curvatures, $\left.n=4\right)$, (Figs 31-64). The forward stroke of the PF took a longer time (8-16 ms) than the recovery stroke (5-8 ms) (Figs 47, 64, Movie S4). It also became clear that the AF waveform was not affected by the PF waveform in chemotactic male gametes (Figs 47, 64).

\section{Discussion}

Brown algal swarmers (heterokont algae, Stramenopiles) including gametes and zoospores have morphologically and functionally heterogeneous flagella. This is quite different from the cases of the green alga, Chlamydomonas, and the sperm of marine invertebrates. In this study, swimming velocities of E. siliculosus male and female gametes were about $200-240 \mu \mathrm{m} \mathrm{s}^{-1}$ in the free-swimming case and $130-150 \mu \mathrm{m} \mathrm{s}^{-1}$ in 
thigmotactic-swimming one. This value was similar to the previous observation using 16 mm film (Geller \& Müller, 1981). Although the number, length and beat frequency of flagella are diverse, swimming speeds of gametes or sperm in aquatic organisms are not so variable. Some of these values are $174 \mu \mathrm{m} \mathrm{s}^{-1}$ in Chlamydomonas reinhardtii $\mathrm{P}$. A. Dangeard (Goodenough, 1983), $165 \mu \mathrm{m} \mathrm{s}^{-1}$ in sea urchin (Wood et al., 2005), 276 $\mu \mathrm{m} \mathrm{s}^{-1}$ in ascidians (Yoshida et al., 2002), and $375 \mu \mathrm{m} \mathrm{s}^{-1}$ in starfish (Shiba et al., 2006).

Thigmotactic-swimming gametes of E. siliculosus did not show helical rotations of their cell body and they displayed only two-dimensional waveforms of the AF. While, free-swimming gametes frequently showed helical rotations of their cell body and they displayed two- and three-dimensional waveforms of the AF. It was mathematically shown by Chwang \& Wu (1971) and Keller \& Rubinow (1976) that three-dimensional (helical) flagellar movement induces rotation of the cell. Helical rotations of Ectocarpus gametes would be induced by a combination of several complex AF waveforms as the suggestions of Geller and Müller (1981). Although there is no significant difference in beat frequency of the AF between free- and thigmotactic-swimming gametes, swimming velocities between them were different. AF amplitude also did not seem to be effective to the gamete swimming speed. An increase in swimming speed in free-swimming gametes may be caused by helical rotation of the gamete cell body. While, Ishijima (2012) examined the movement characteristics of the sperm and flagella from a lancelet and 35 species of fishes using high-speed video microscopy. He concluded that flagellar beat frequency was most effective to the swimming speed, neither the flagellar wavelength nor amplitude. 
After Müller $(1967,1971)$ first discovered the brown algal pheromone 'ectocarpene' in Ectocarpus siliculosus, many other sex pheromones in brown algae were identified (see the reviews of Maier \& Müller, 1986 and Maier, 1993). As a result of these studies, detailed observations of the behaviour of male and female gametes during the fertilization process of E. siliculosus were carried out. Ectocarpus male and female gametes are morphologically isomorphic. Female gametes settle down on the substratum quicker than male gametes. When settled, the female gametes begin to secret the sex pheromone. However, the locomotion and flagellar waveforms of male and female gametes were observed to be almost same after adding them into the chamber.

During chemotaxis in the presence of sex pheromone, the AF deflection angle of male gametes significantly changed from the control with decreasing beat frequency of the AF. Geller \& Müller (1981) showed a correlation between the average deflection angle of the AF and the radius of the track. In addition to their results, we clarified that the deflection angle of the AF was significantly changed when male gametes altered their swimming pattern from highly linear paths to small or medium circular paths. However, the deflection angle did not significantly change when the gametes switched from medium to small circular paths. Therefore, it was suggested that changing the swimming paths of male gametes from a highly linear path to a circular path was affected by the AF itself. While, alternating the trajectory from a medium circular to a small circular path was attributed to the PF.

Geller \& Müller (1981) and Maier \& Calenberg (1994) showed that the PF performed fast and unilateral beats, when the cell sensed decreasing concentration of 
pheromone. Unilateral beats of the PF were usually observed for reorientation of male gametes towards the sex pheromone source (settled female gametes). In this study, it became clear that, regardless of unilateral beating of the PF, the waveform of the AF remained stable (Figs 47, 64). It was suggested that the AF and PF would move independently in the presence of sex pheromone.

Matsunaga et al. (2010) showed the rapid lateral beats of the PF during phototactic-orientation of gametes in the brown alga, Scytosiphon lomentaria. Those studies reveal that the forward stroke of PF took a longer time (6-8 ms) than the recovery stroke (2-4 ms). When the gametes show phototactic turning, AF undulation ceased. Therefore, AF motion during phototactic turning of gametes is clearly different from the case of the chemotactic one, although unilateral beat patterns of the PF seem to be similar.

It was revealed that the AF plays a role in changing gamete locomotion from freeto thigmotactic-swimming and also from straight to circular swimming. Moreover, the AF changes the flagellar asymmetry and deflection angle during these steps but it does not change these properties in the presence of the sex pheromone. The PF shows irregular changes in the deflection angle and large bending are dependent on the concentration gradient of the sex pheromone. The exact timing and mechanism of large unilateral beats in the PF during chemotaxis remain unclear. In the ascidian, Yoshida \& Yoshida (2011) report that sperm attractants appear to induce $\mathrm{Ca}^{2+}$ entry from extracellular spaces into sperm cells. This increases the intracellular $\mathrm{Ca}^{2+}$ concentration which mediates the beating patterns of sperm flagella. This increase in $\mathrm{Ca}^{2+}$ 
concentration results in the observed chemotactic turn. Maier and Calenberg (1994) report on the effects of calcium on flagellar movements of E. siliculosus male gametes during chemotaxis. They surveyed several calcium antagonists and channel blockers including Lanthanum, Ruthenium Red, Verapamil, Nifedipine, and Trifluoperazine, and showed that: 1) the asymmetric bending of the AF of male gametes will be strongly inhibited by $\left.\mathrm{La}^{3+}, 2\right)$ Nefedipine might interact with $\mathrm{Ca}^{2+}$ channels which are involved in the action of PF, and 3) Trifluoperazine might bind to and activate the pheromone receptors in Ectocarpus via hydrophobic interactions. Recently, Fu et al. (2014) conducted a proteomics analysis of flagella of the brown alga, Colpomenia bullosa (Saunders) Yamada suggested that $8 \%$ of the 495 flagellar proteins were related to proteins with calcium-binding function. Further investigation on the roles of calcium in flagellar bending using a high-speed camera will clarify the characteristic beat patterns of the heterogeneous AF and PF of brown algal gametes during chemotaxis.

\section{Acknowledgement}

This study was partially supported by the Japanese Association for Marine Biology (25-55, 26-56). 


\section{Reference}

Baba, S.A. \& Mogami, Y. (1985). An approach to digital image analysis of bending shapes of eukaryotic flagella and cilia. Cell Motility, 5: 475-489.

Bouck, G.B. (1969). Extracellular microtubules: the origin, structure, and attachment of flagellar hairs in Fucus and Ascophyllum antherozoids. Journal of Cell Biology, 40: 446-460.

Andersen, R.A. (2004). Biology and systematics of heterokont and haptophyte algae. American Journal of Botany, 91: 1508-1522.

Chwang, A.T. \& Wu, T.Y. (1971). A note on the helical movement of micro-organisms. Proceedings of the Royal Society of London. Series B, Biological Sciences, 178: $327-346$.

Clayton, M. N. (1989). Brown algae and chromophyte phylogeny: In The Chromophyte Algae: problems and perspectives. (Green, J.C., Leadbeater, B.S.C.\& Diver, W.L. editors), The Systematics Association Special, 38: 230-253. Clarendon Press, Oxford, UK.

Geller, A. \& Müller, D.G. (1981). Analysis of the flagellar beat pattern of male Ectocarpus siliculosus gametes (Phaeophyta) in relation to chemotactic stimulation by female cells. Journal of Experimental Biology, 92: 53-66.

Gibbons, I.R. (1981). Cilia and flagella of eukaryotes. Journal of Cell Biology, 91(suppl): 107s-124s.

Goodenough, U.W. (1983). Motile detergent-extracted cells of Tetrahymena and Chlamydomonas. Journal of Cell Biology, 96: 1610-1621. 
Holwill, M.E.J. \& Sleigh, M.A. (1967). Propulsion by hispid flagella. Journal of Experimental Biology, 47: 267-276.

Inaba, K. (2003). Molecular architecture of the sperm flagella: molecules for motility and signaling. Zoological Science, 20: 1043-1056.

Ishijima, S. (2012). Comparative analysis of movement characteristics of lancelet and fish spermatozoa having different morphologies. The Biological Bulletin, 222: 214-221.

Jahn, T.L., Landman, M.D. \& Fonseca, J.R. (1964). The mechanism of locomotion of flagellates. II. Function of the mastigonemes of Ochromonas. Journal of Protozoology, 11: 291-296.

Kaupp, U.B., Kashikar, N.D. \& Weyand, I. (2008). Mechanisms of sperm chemotaxis. Annual Reviews Physiology, 70: 93-117.

Kawai, H., Müller, D.G., Folster, E. \& Häder, D.P. (1990). Phototactic responses in the gametes of the brown alga, Ectocarpus siliculosus. Planta, 182: 292-297

Kawai, H. \& Kreimer, G. (2000). Sensory mechanisms: Light perception and taxis in algae. In The flagellates: Unity, Diversity and Evolution (Leadbeater, B. \& Green, J., editors), 124-146. Taylor and Francis, London and New York.

Keller, J.B. \& Rubinow, S.I. (1976). Swimming of flagellated microorganisms. Biophysical Journal, 16: 151-170.

Maier, I. (1993). Gamete orientation and induction of gametogenesis by pheromone in algae and plants. Plant, Cell \& Environment, 16: 891-907. 
Maier, I. (1995). Brown algal pheromones. In Progress in Phycological Research (Round, F. E. \& Chapman, D. J., editors), 11: 51-102. Biopress, Bristol, UK.

Maier, I., \& Müller, D.G. (1986). Sexual pheromones in algae. Biological Bulletin, 170: 145-175.

Maier, I. \& Calenberg, M. (1994). Effect of extracellular $\mathrm{Ca}^{2+}$ and $\mathrm{Ca}^{2+}$ antagonists on the movement and chemoorientation of male gametes of Ectocarpus siliculosus (Phaeophyceae). Botanica Acta, 107: 451-460.

Matsunaga, S., Uchida, H., Iseki, M., Watanabe, M. \& Murakami, A. (2010). Flagellar motions in phototactic steering in a brown algal swarmer. Photochemistry and Photobiology, 86: 374-381.

Miller, R.L. (1975). Chemotaxis of the spermatozoa of Ciona intestinalis. Nature, 254: 244-245.

Miller, R.L. (1977). Chemotactic behavior of the sperm of chitons (Mollusca: Polyplacophora). Journal of Experimental Zoology, 202: 203-211.

Miller, R. L. \& Brokaw, C. J. (1970). Chemotactic turning behaviour of Tubularia spermatozoa. Journal of Experimental Biology, 52: 699-706.

Mitchell, D.R. (2000). Chlamydomonas flagella. Journal of Phycology, 36: 261-273.

Müller. D.G. (1967) Ein leicht flüchtiges Gyno-Gamon der Braunalge Ectocarpus siliculosus. Naturwissenschaften, 54: 496-497.

Müller, D.G. (1978). Locomotive responses of male gametes to the species-specific sex attractant in Ectocarpus siliculosus (Phaeophyta). Archiv für Protistenkunde, 120: 371-377. 
Müller, D.G. \& Falk, H. (1973). Flagellar structure of the gametes of Ectocarpus siliculosus (Phaeophyta) as revealed by negative staining. Archiv für Mikrobiologie, 91: 313-322.

Müller, D.G., Jaenicke, L., Donike, M. \& Akintobi, T. (1971). Sex attractant in a brown alga: chemical structure. Science, 171: 815-817.

O' Kelly, C.J. (1989). The evolutionary origin of the brown algae: Information from studies of motile cell structure. In The Chromophyte Algae: problems and perspectives. (Green, J.C., Leadbeater, B.S.C.\& Diver, W.L. editors), The Systematics Association Special, 38: 256-278. Clarendon Press, Oxford, UK.

Provasoli, L. (1968). Media and prospects for the cultivation of marine algae. In Cultures and collections of algae (Watanabe, A. \& Hattori, A., editors), 63-75. Japanese Society of Plant Physiology, Hakone.

Shiba, K., Tagata, T., Ohmuro, J., Mogami, Y., Matsumoto, M., Hoshi, M. \& Baba, S. A. (2006). Peptide-induced hyperactivation-like vigorous flagellar movement in starfish sperm. Zygote, 14: 23-32.

Shiba, K., Baba, S. A., Inoue, T., \& Yoshida, M. (2008). $\mathrm{Ca}^{2+}$ bursts occur around a local minimal concentration of attractant and trigger sperm chemotactic response. Proceedings of the National Academy of Sciences of the United States of America, 105: 19312-19317.

Wood, C.D., Nishigaki, T., Furuta, T,, Baba, S. A., Darszon, A. (2005) Real-time analysis of the role of $\mathrm{Ca}^{2+}$ in flagellar movement and motility in single sea urchin sperm. Journal of Cell Biology, 169: 725-731. 
Yoshida, M., Murata, M., Inaba, K., \& Morisawa, M. (2002). A chemoattractant for ascidian spermatozoa is a sulfated steroid. Proceedings of the National Academy of Sciences of the United States of America, 99: 14831-14836.

Yoshida, M. \& Yoshida, K. (2011). Sperm chemotaxis and regulation of flagellar movement by $\mathrm{Ca}^{2+}$. Molecular Human Reproduction, 17: 457-465. 


\section{Figure legends}

Figs 1-3. The trajectories of free- and thigmotactic-swimming male gametes in $E$. siliculosus. 1, 2. The swimming trajectories of free-swimming male gametes (1) and thigmotactic-swimming male gametes (2) are obtained by image processing during a period of 2s. 3. Frequency of the swimming path curvatures (absolute value) of thigmotactic-swimming male gametes $(\mathrm{n}=115)$. Scale bars $=100 \mu \mathrm{m}$.

Figs 4-13. The anterior flagellar (AF) waveforms of free- and thigmotactic-swimming male gametes. 4-6. A typical flagellar waveform of free-swimming gametes (symmetrical waveform of the AF) recorded at 1.6-ms intervals. 7-9. A typical flagellar waveform of thigmotactic-swimming male gametes recorded at 1.6-ms intervals. 10, 11 . Trace of AF waveforms in free-swimming male gametes (10) and thigmotactic-male gametes (11). Twenty frames were chosen every $1.6 \mathrm{~ms}$ and traces of the AF were merged. Black bars show $5 \mu \mathrm{m}$ reference line and grey dots indicate the anterior end of the cell body. 12. Flagellar asymmetric indices of free- and thigmotactic- swimming male gametes. I. Basal region of the AF; II. Middle region; III. Tip region; IV. Whole region. Free- vs. thigmotactic-swimming male gametes, $* \mathrm{P}<0.001$. 13. Average value of the deflection angle at $8 \mu \mathrm{m}$ from the tip of the AF. I. Free-swimming male gametes; II. Thigmotactic-male gametes. Free- vs. thigmotactic-swimming male gametes, $* \mathrm{P}<0.001$. Scale bars $=5 \mu \mathrm{m}$.

Figs 14-16. The posterior flagellar (PF) waveforms of free- and thigmotactic-swimming male gametes. 14, 15. Trace of the PF waveforms in free- (14) and thigmotactic-swimming male gametes (15). Twenty forms were chosen every $1.6 \mathrm{~ms}$ 
and traces of the PF were merged. Black bars show $5 \mu \mathrm{m}$ reference line and gray dots indicate the anterior end of the cell body. 16. The deflection angle at $1 \mu \mathrm{m}$ from the tip of PF is plotted against time.

Figs 17-19. The trajectories of chemotactic male gametes. 17, 18. The swimming trajectories of chemotactic gametes are obtained by image processing during the period of 2s. 17. Free-swimming male gametes on the upper region from settled female gametes. 18. Thigmotactic-swimming male gametes near settled female gametes. White arrowheads shows settled female gametes. White double arrowheads shows the swimming path of 20. 19. Frequency of the swimming path curvatures $(n=115)$. Scale bars $=100 \mu \mathrm{m}$.

Figs 20-29. The AF beat patterns of chemotactic male gametes. 20-25. Trajectory (20) and typical flagellar waveforms were recorded at 1.6-ms intervals (21-25). 26. The AF asymmetric indices of chemotactic male gametes. I. Base region; II. Middle region; III. Tip region; IV. Whole region. Control vs. Chemotactic gametes, ${ }^{* *} \mathrm{P}<0.05 .27$. Asymmetric indices of tip region in the AF plotted against path curvatures. 28. Average value of deflection angle at $8 \mu \mathrm{m}$ from the tip of AF. I. Control; II. Chemotactic gametes. Control vs. chemotactic gametes, ${ }^{*} \mathrm{P}<0.001$. 29. Average value of deflection angles at $8 \mu \mathrm{m}$ from the tip of AF plotted against path curvatures. Scale bars $=10 \mu \mathrm{m}$. Fig. 30. A trajectory and PF bending pattern of a chemotactic male gamete $\left({ }^{\top}\right)$ near female gamete (우). White squares show the timing of lateral PF beating of male gamete. Gray squares show the motionless PF. A-F show detailed images of male 
gametes at each square, respectively. Arrows indicate the position of the PF. Scale bar = $20 \mu \mathrm{m}$ in 30, and $10 \mu \mathrm{m}$ in 30A-F.

Figs 31-64. Two patterns of lateral beats of the PF in chemotactic male gametes. PF bent for a while (31-47) and PF repeated beating (48-64). Images were recorded at 1.6-ms intervals under the same conditions (45-47 were analysed from 31-44, and 62-64 were analysed from 48-61). 45, 62. Trajectories of chemotactic male gametes. Arrows indicate the timing of unilateral beats of PF. 46, 63. Trace of AF and PF waveforms. Twenty forms were chosen every $1.6 \mathrm{~ms}$ and merged. Black bars show $5 \mu \mathrm{m}$ reference line and grey dots indicate the anterior end of cell body. 47, 64. Deflection angles at 8 $\mu \mathrm{m}$ from the tip of the AF and at $1 \mu \mathrm{m}$ from the tip of the PF are plotted. Arrows indicate the timing of unilateral beats of PF in $\mathbf{4 5}$ and $\mathbf{6 2}$. Scale bars $=10 \mu \mathrm{m}$. Figs S1, 2. Definitions of the terms. 1. Definition of the swimming path curvature $(\Gamma)$ and the flagellar curvature $(\gamma)$. Blue dots and lines show the center of cell body and zigzag trajectory of the cell body. 2. Definition of the deflection angle $(\theta)$.

Figs S3-5. AF curvature plotted against distance from near the base of the flagellum. The basal part of AF was hidden by the cell body, therefore the distance of the base of AF was obtained by adding 4 um (as the half width of the cell body) to the AF length AF which was detected under microscopy. Data from 20 waveforms were merged. 3. Free-swimming male gametes. 4. Thigmotactic-swimming male gametes. 5. Chemotactic-male gametes.

Fig. S6. Distribution of deflection angles of the PF of the control $(n=148$ plots from 5 male gametes) and chemotactic-male gametes ( $n=148$ plots from 4 male gametes). 
Movie S1. Free-swimming male gametes.

Movie S2. Thigmotactic-swimming male gametes.

Movie S3. Chemotactic-male gametes.

Movie S4. Rapid lateral beats of the PF of male gametes. 


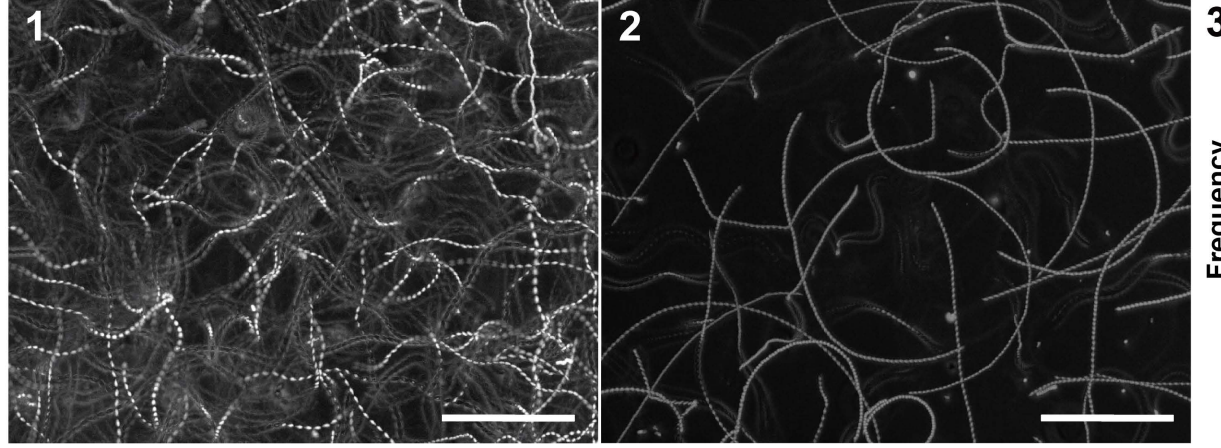
Path curvature $\left(\mu \mathrm{m}^{-1}\right)$ 
1415

16
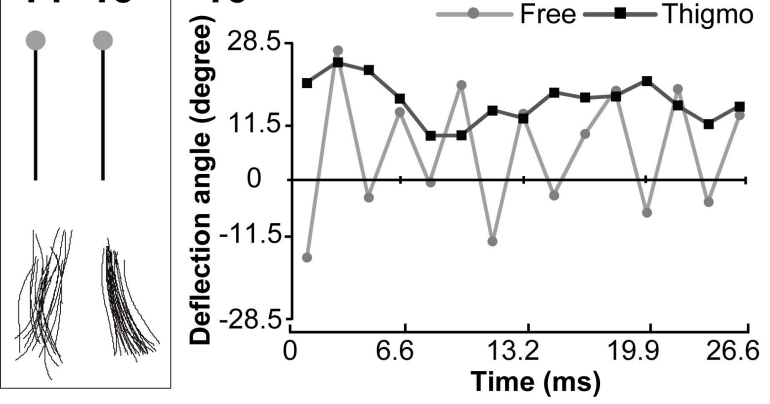


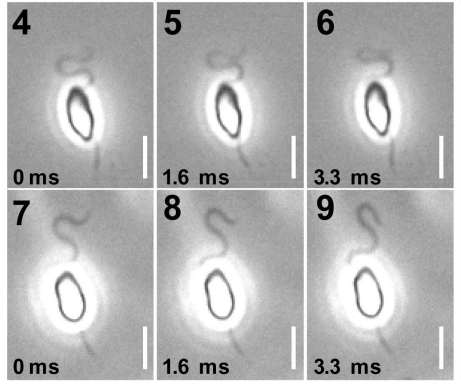

12

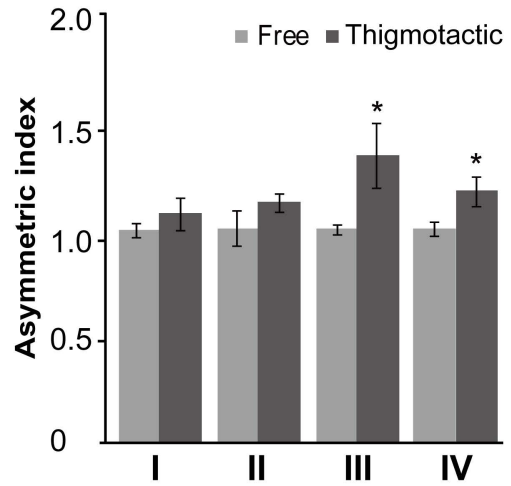

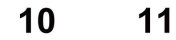

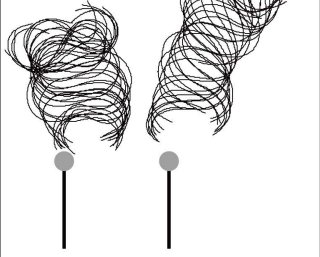

13

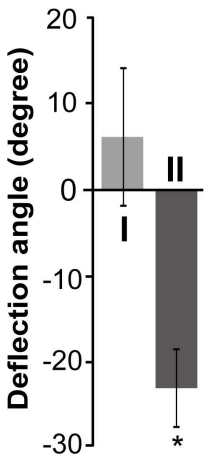



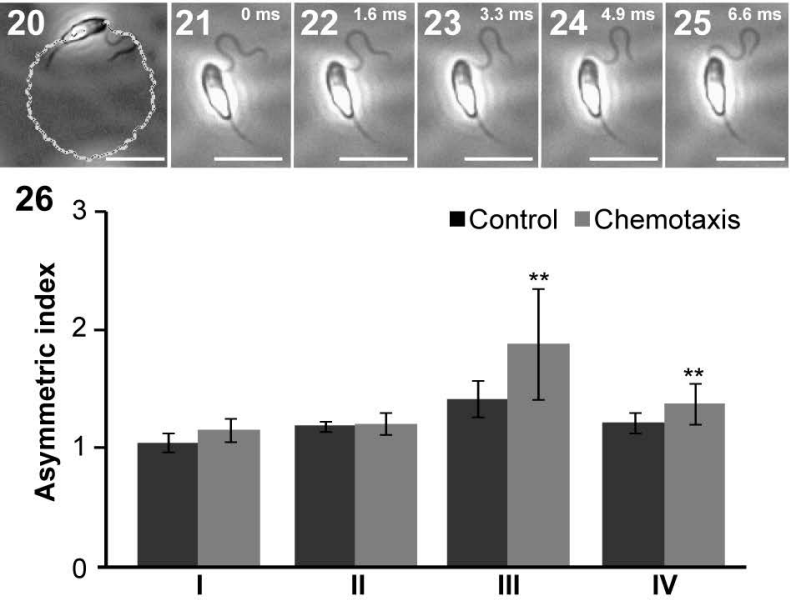

2

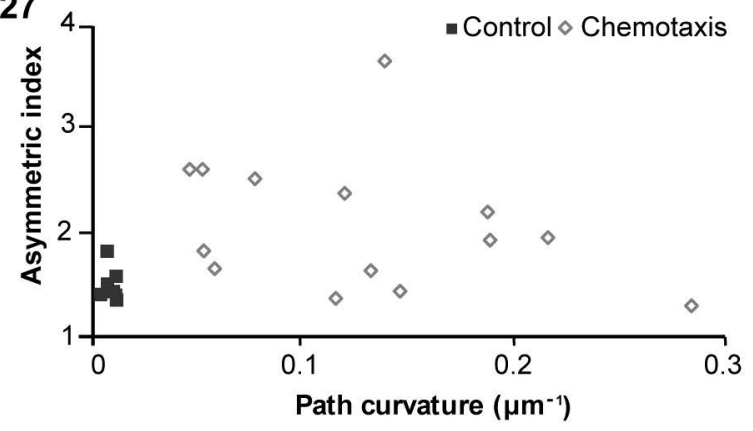

28

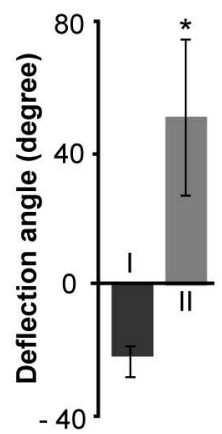

29

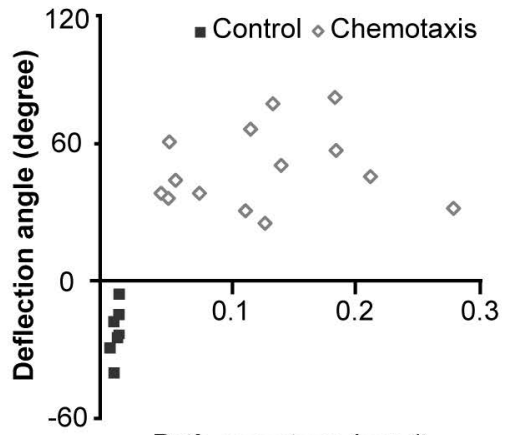

Path curvature $\left(\mu \mathrm{m}^{-1}\right)$ 

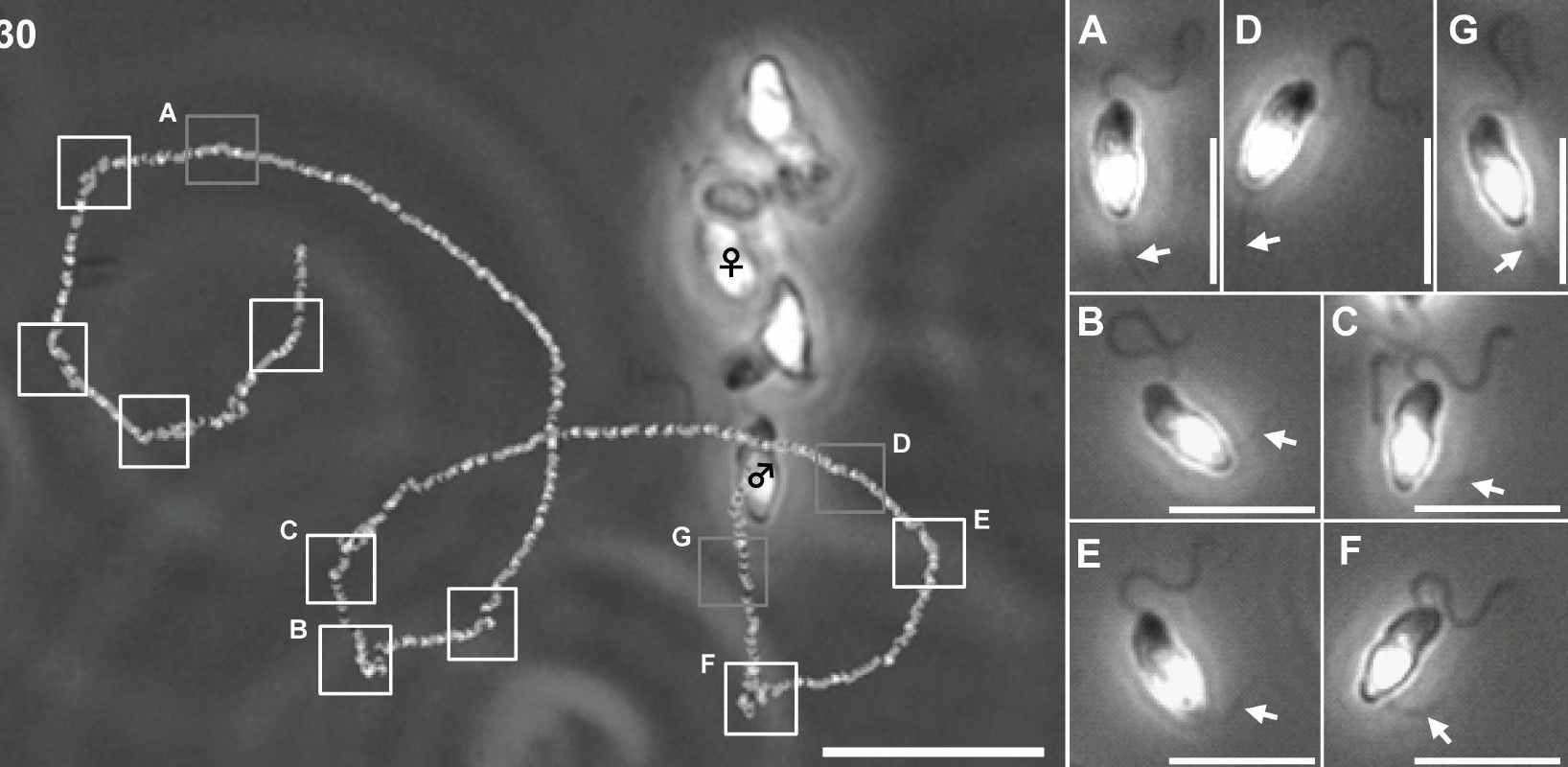

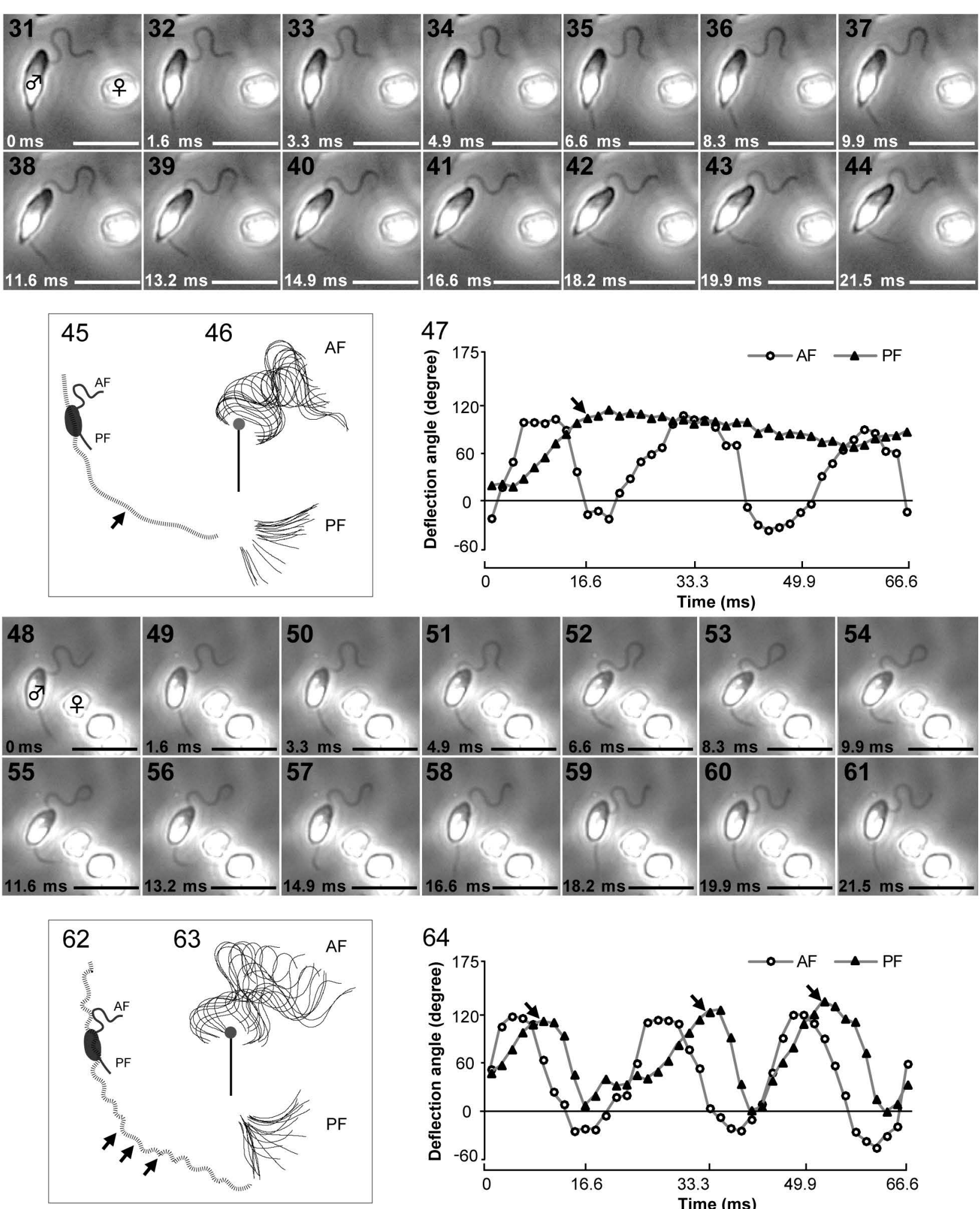

64

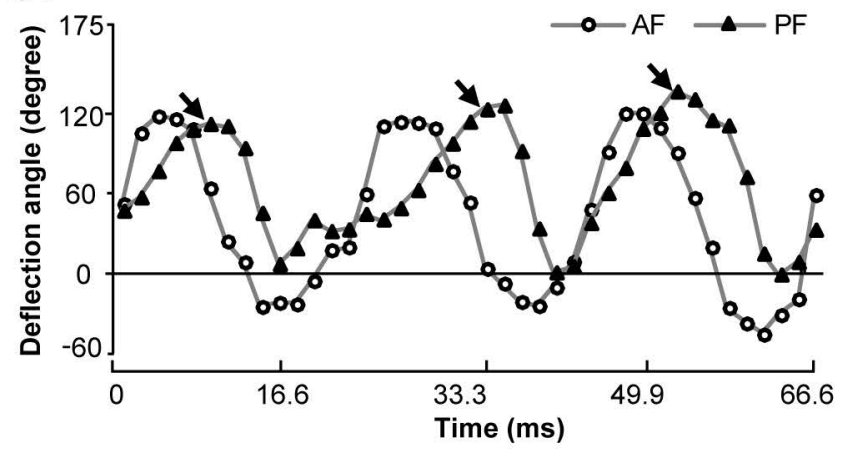



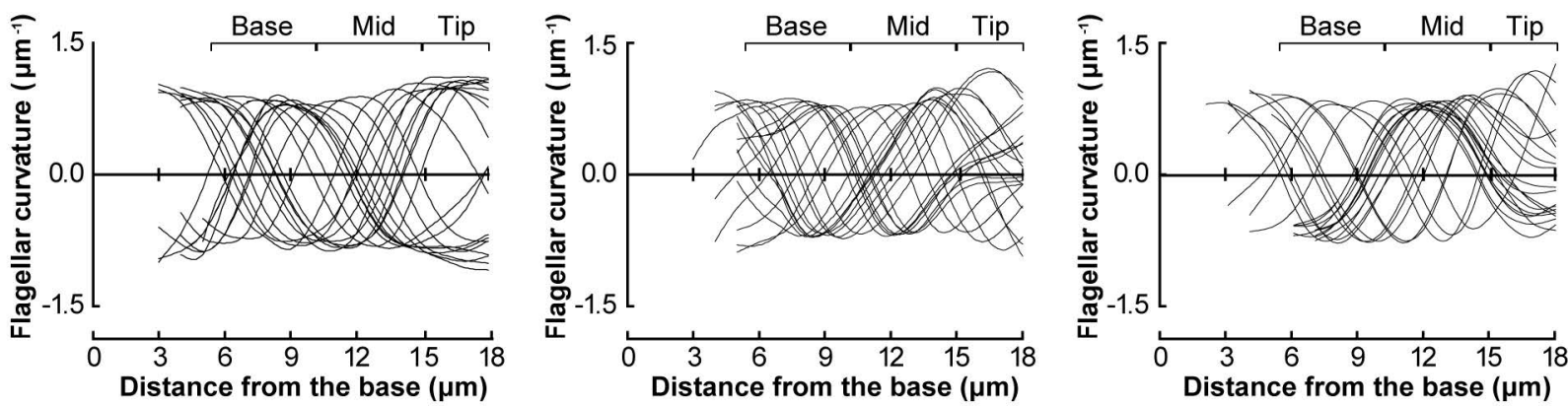
